DOI: http://dx.doi.org/10.15517/rce.v35i2.31746

\title{
ESTIMACIÓN DE LA TASA DE INTERÉS REAL NEUTRAL PARA COSTA RICA
}

\author{
Evelyn Muñoz-Salas ${ }^{1}$ \\ Adolfo Rodríguez-Vargas ${ }^{2}$
}

Recibido: 30/08/2017

Aprobado: 10/10/2017

\begin{abstract}
RESUMEN
Un banco central que utilice la tasa de interés como instrumento de control monetario requiere contar con una estimación de la tasa de interés real neutral (TIRN) como referencia para tomar las decisiones de política conducentes a lograr su objetivo de inflación. Este documento aborda por primera vez la estimación de la tasa de interés real neutral, utilizando datos de una tasa de política monetaria para Costa Rica, establecida en junio del 2011 por el Banco Central de Costa Rica como su instrumento de control monetario. Considerando información mensual para el período 2009-2015 y combinando las estimaciones provenientes de diversas metodologías, la TIRN para Costa Rica se estima en torno a 1,4\%.
\end{abstract}

PALABRAS CLAVE: POLÍTICA MONETARIA, INFLACIÓN, VARIABLES NO OBSERVABLES. CLASIFICACIÓN JEL: E12, E31, E52.

\section{ABSTRACT}

A central bank that uses the interest rate as monetary policy instrument requires an estimation of the neutral real interest rate (NRIR) that allows it to define the monetary policy stance to achieve its inflation target. This paper estimates the NRIR from actual values of the Monetary Policy Rate (TPM) for Costa Rica for the first time. This rate was established by the Central Bank of Costa Rica as its instrument for monetary control on June 2011. On the basis of monthly data from 2002-2015 and combining estimates from several methodologies, we estimate the neutral real interest rate for Costa Rica to be around $1,4 \%$.

KEYWORDS: MONETARY POLICY, INFLATION, UNOBSERVABLE VARIABLES.

JEL CLASIFICATION: E12, E31, E52.

1 Banco Central de Costa Rica, Departamento de Investigación Económica; Código Postal 10058-1000. San José Costa Rica; munozse@bccr.fi.cr

2 Banco Central de Costa Rica, Departamento de Investigación Económica; Código Postal 10058-1000. San José Costa Rica; rodriguezva@bccr.fi.cr 


\section{INTRODUCCIÓN}

El Banco Central de Costa Rica (BCCR) se encuentra en un proceso de modernización de su política monetaria, para mantener la estabilidad interna de la moneda nacional, tal como le confiere su Ley Orgánica (Ley 7558, 1995). El BCCR interpreta este objetivo como lograr y mantener una inflación baja y estable, coherente con la de los principales socios comerciales del país, y así lo comunica al público en los programas macroeconómicos (Banco Central de Costa Rica, 2016).

Uno de los hitos más visibles de este proceso de modernización fue establecer la tasa de política monetaria (TPM) como instrumento de control monetario ${ }^{3}$. En junio del 2011, se definió la TPM como el valor central del corredor de tasas de interés en el Mercado integrado de liquidez ${ }^{4}$ (MIL). Con este instrumento se busca influir en el costo marginal de la liquidez de los intermediarios financieros $y$, de esta forma, transmitir el tono de la política monetaria al resto de tasas del sistema financiero.

Esta decisión se tomó en un contexto de flexibilidad cambiaria creciente, lo que le brinda una independencia cada vez mayor al BCCR en el uso de este instrumento, pues en febrero del 2015 se adoptó un régimen cambiario de flotación administrada, luego de casi nueve años de mantener una banda cambiaria que se había anunciado como transitoria en el 2006.

Para evaluar la postura de la política monetaria y apoyar la toma de decisiones en términos de tasa de interés con el fin de cumplir con la meta de inflación, se requiere contar con una estimación de la tasa de interés real neutral (Amato, 2005). El término tasa de interés real neutral (TIRN) o tasa de interés real natural se refiere al nivel de tasa de interés real que se asocia con una política monetaria que no es ni expansiva ni contractiva (Fuentes, 2008).

Dos antecedentes de estimación de la TIRN para Costa Rica son Muñoz y Tenorio (2007) y Segura y Vindas (2012). En ambos estudios del BCCR, se empleó un indicador de costo promedio de los recursos en el sistema financiero como una aproximación de la postura de la política monetaria, debido a la ausencia de una TPM, en el primer caso, y a la escasa información histórica sobre ella, en el otro. Organismos internacionales como la Organización para la Cooperación y el Desarrollo Económico (OCDE, 2016) y el Fondo Monetario Internacional (FMI, 2016) también han estimado esta variable no observable, con las mismas limitaciones en lo referente al indicador de tasa de interés.

En vista de estas consideraciones, esta investigación aproxima la tasa de interés real neutral para Costa Rica mediante metodologías estructurales utilizando por primera vez información de una tasa de política monetaria.

\section{La tasa de interés real neutral}

Generalmente, se le reconoce al economista sueco Knut Wicksell el haber introducido en 1898 el concepto de la tasa de interés natural (o neutral) en contraposición a la tasa de interés de mercado. Wicksell resumió la idea de la tasa natural como "la tasa para préstamos que es neutral hacia los precios de las mercancías, y que no tiende a incrementarlos ni a disminuirlos” (Wicksell, 1938, pp.106, traducción propia).

Su teoría se basa en la diferencia entre el costo del crédito y el producto marginal del capital. Wicksell postuló que si la tasa de interés de mercado es inferior a la tasa natural de retorno del capital, es decir, al producto marginal del capital, eso incentivaría a los empresarios a tomar préstamos a la tasa de

3 El encaje mínimo legal constituye uno de los principales instrumentos de política monetaria. No obstante, desde el 2005 se encuentra en el máximo que la Ley Orgánica del BCCR permite (Ley 7558, 1995).

4 El MIL es una plataforma de negociación organizada por el BCCR, en la que participan entidades financieras debidamente autorizadas, intercambiando recursos líquidos conforme sus requerimientos. Este mercado permite al BCCR una gestión eficiente de la liquidez sistémica, inyectando o drenando recursos y, de esa forma, ubicar el costo de la liquidez en torno a la TPM (Banco Central de Costa Rica, 2011). 
mercado para acumular capital, lo que eventualmente elevaría la demanda por todos los bienes y llevaría a un aumento en el nivel general de precios o, lo contrario ocurriría en el caso de una tasa de mercado más alta que la natural. El aumento o la disminución de precios se mantendría en tanto persistiera la diferencia entre la tasa natural de retorno del capital y la tasa de interés.

Para Wicksell, la posibilidad de mantener estabilidad en los precios dependería, todo lo demás constante, "del mantenimiento de una cierta tasa de interés para préstamos, y que una discrepancia permanente entre la tasa vigente $y$ [la tasa natural] ejerce una influencia progresiva $y$ acumulativa sobre los precios" (Wicksell, 1938, pp. 120-21, traducción propia) ${ }^{5}$.

Wicksell (1938) argumentó que es inútil tratar de demostrar alguna relación directa entre movimientos absolutos de la tasa de interés y los cambios en precios, sino que dicha discusión es significativa solamente si se realiza en sentido relativo comparando las tasas de interés natural y de mercado. Así, la idea de una tasa de interés "alta" (o "baja") sería esencialmente un concepto relativo para cuya valoración sería necesario contar con información sobre la tasa natural. Ahora bien, ya desde su primera formulación del concepto, Wicksell reconoció que la tasa natural no es observable ni debe esperarse que sea constante, pues cambiaría de acuerdo con el retorno del capital, que estaría influido por la eficiencia de la producción, por la disponibilidad de capital físico y líquido, y en la oferta de capital y tierra, entre otros. Por ende, postuló que una coincidencia exacta de ambas tasas es poco probable y que su discusión en general se refería a un "nivel general medio".

Si bien en su Tratado sobre el dinero (1930), J. M. Keynes esbozó una teoría para la determinación de una única tasa de interés, a la que llamó tasa natural, la cual correspondía a la tasa que igualaba la "tasa de ahorro y la tasa de inversión" (en la terminología de esa obra), para la publicación de su Teoría General (1936) su pensamiento había evolucionado para rechazar explícitamente esa idea ${ }^{6}$. En esa obra, Keynes argumentó que la preferencia por liquidez junto con la oferta de dinero determina el nivel de la tasa de interés, y que la existencia de múltiples equilibrios correspondientes a diferentes niveles de ingreso y empleo estaría asociada con diferentes niveles de tasa natural de interés.

En décadas recientes, el concepto de tasa de interés neutral ha vuelto a recibir atención dentro de la teoría neokeynesiana. El aporte definitivo para cimentar este concepto como central a la macroeconomía moderna es el libro de Woodford "Interés y precios: fundamentos para una teoría de la política monetaria" (2003). Tomando como marco de análisis un modelo de equilibrio general dinámico y estocástico básico compuesto por una ecuación intertemporal de Euler, una curva de Phillips neokeynesiana y una regla de tasa de interés tipo Taylor (1993), Woodford demuestra que es posible obtener una tasa natural de interés conceptualmente equivalente a la propuesta por Wicksell. Elaborando de manera formal un argumento propuesto ya por el economista sueco, Woodford muestra que, en este contexto, la tasa natural es una función de variables fundamentales como las preferencias de los consumidores o choques de productividad $y$, por tanto, es invariable a las decisiones de política. Para Woodford, al igual que para Wicksell, este valor natural es el que prevalecería si no hubiese rigideces nominales ${ }^{7}$.

Crucialmente, en el capítulo 4 de "Interés y precios", Woodford muestra que es posible realizar política monetaria de estabilización de precios únicamente en términos de una regla para la tasa de interés, sin considerar la oferta de dinero, y que dicha política de estabilización es deseable desde el

5 Dado que el esquema de patrón oro imperante en la época hacía poco probable la existencia de inflaciones elevadas, la discusión wickselliana de la tasa de interés neutral se debe entender como referida a tasas reales.

6 En el capítulo 17, Keynes afirma abiertamente: "Ya no mantengo la opinión de que el concepto de una tasa 'natural' de interés, que previamente parecía una idea muy promisoria, tenga algo muy útil o significativo que contribuir al análisis” (Keynes, 1936, p. 153, traducción propia).

7 Esta definición es esencialmente la que utilizan autores como Galí (2002): “...la tasa de interés real que prevalecería en la economía si no existiera ninguna rigidez nominal”; Laubach y Williams (2003): “...la tasa de interés real de corto plazo coherente con un producto convergente al potencial, donde el nivel del producto potencial es coherente con una tasa de inflación estable"; y BrzozaBrzezina (2003): "Nivel de la tasa de interés real que hará a la política monetaria neutral y ayuda a estabilizar la inflación...". 
punto de vista de bienestar. Estos resultados han provisto justificación formal teórica a la manera en que se conduce la política monetaria en buena parte de los bancos centrales en la actualidad, particularmente los que han adoptado un régimen de metas de inflación.

En la práctica vigente, mediante una regla de política tipo Taylor, la TIRN constituye la referencia que caracteriza la postura de la política monetaria. Cuando la tasa de política real se ubica por debajo del nivel de la tasa real neutral, la política monetaria es laxa o expansiva, lo cual podría implicar presiones inflacionarias en el futuro. Caso contrario cuando la tasa de política monetaria real excede la TIRN indicando una postura de política contractiva con el objetivo de controlar presiones inflacionarias ${ }^{8}$.

Los modelos macroeconómicos neokeynesianos utilizados por buena parte de los bancos centrales incorporan el concepto de tasa real neutral y lo asocian con la tasa que es coherente con el equilibrio de pleno empleo en la economía, y ausencia de presiones inflacionarias (Fuentes, 2008).

Sin embargo, para utilizar la TIRN como referencia en la formulación de la política monetaria es necesario contar con estimaciones de su valor, lo que se complica por el hecho de ser una variable no observable, que además varía en función de factores estructurales de la economía.

\section{Antecedentes para Costa Rica}

Considerando información para el período 1991 al 2006, Muñoz y Tenorio (2007) realizaron cuatro estimaciones para la tasa de interés real neutral: una estimación ad-hoc que consiste en la tasa de interés real promedio vigente durante un período de inflación estable; una estimación de la tendencia de largo plazo mediante el filtro univariado de Hodrick-Prescott; una aproximación basada en el criterio de paridad descubierta de tasas de interés en términos reales y otra a partir de un modelo semiestructural estimado mediante el filtro de Kalman ${ }^{9}$, siguiendo el enfoque propuesto por Laubach y Williams (2003).

Por su parte, Segura y Vindas (2012) realizaron sus estimaciones a partir de información mensual para el período 2001-2011, lo que significa que cubrieron al menos tres años del período de vigencia de la banda cambiaria. Además de las metodologías empleadas por Muñoz y Tenorio (2007), incorporaron una estimación con un modelo estructural de vectores autorregresivos (SVAR) propuesta por Brzoza-Brzezina $(2002,2006)$.

El FMI (2016) y la OCDE (2016) han generado estimaciones de la TIRN en el marco de sus evaluaciones del estado de la economía costarricense. El FMI utiliza un modelo semiestructural neokeynesiano, un modelo de equilibrio general, un modelo monetario de carácter prospectivo y una regla de Taylor aumentada por expectativas, que además es el método utilizado por la OCDE.

De acuerdo con las estimaciones disponibles de la TIRN para Costa Rica pasó de alrededor de 2,9\% entre el 2001 y 2006 (Muñoz y Tenorio), a un 1,9\% de acuerdo con Segura y Vindas (2012), esta última estimación incorpora cinco años de información adicional, específicamente el período de la crisis financiera internacional de los años $2008-2009^{10}$. Debe recordarse que ambos estudios utilizan la Tasa básica pasiva como el indicador de política del Banco Central, ante la ausencia de una tasa de política monetaria coherente para un período extenso.

Las estimaciones del FMI y de la OCDE incluyen información hasta el 2015 y consideran datos tanto del régimen cambiario de paridad ajustable como del régimen de flotación administrada, resultan en valores de la tasa de interés real neutral de 1,9\% y 1,6\%, respectivamente ${ }^{11}$.

8 La diferencia entre la tasa de interés de política monetaria y la tasa de interés real neutral da origen al concepto de "brecha en la tasa de interés real" (Amato, 2005).

9 La estimación realizada por estos autores constituye la primewra cuantificación de este parámetro para la economía costarricense.

10 El Cuadro 2 del Anexo resume estos resultados.

11 OCDE utiliza como referencia la TBP para todo el período, en tanto el FMI combina TBP con la TPM a partir de junio del 2011. 


\section{METODOLOGÍA}

Como ya se indicó, al ser la tasa de interés real neutral una variable no observable que no es invariable en el tiempo, su estimación no es sencilla. En vista de ello, una práctica recomendable es recurrir a más de una metodología, esto permite a las autoridades contar con un rango dentro del cual se ubicaría esta variable. Seguidamente, se describen las metodologías empleadas en esta investigación, algunas se basan en teoría económica y otras recurren a enfoques con énfasis estadístico.

El primero de los métodos presentados es la estimación de acuerdo con la condición de paridad descubierta de tasas de interés, considerando una economía pequeña y abierta. Se recurre además a un modelo semiestructural con componentes no observables, $y$ a sistemas de vectores autorregresivos estructurales. Una aproximación válida es la que proviene de métodos univariados que permitan extraer el componente tendencial de una serie de tiempo.

\section{Paridad de tasas de interés}

El concepto de paridad de tasas de interés parte del principio de arbitraje perfecto, según el cual el capital se moviliza entre economías buscando una ganancia en renta, cuyo origen es la diferencia entre los rendimientos.

Considerando este concepto de paridad descubierta de tasas de interés en términos nominales combinado con la ecuación de Fisher (1911), es posible obtener la condición de arbitraje en términos reales, lo cual llevaría a la siguiente expresión ${ }^{12}$ :

$$
r^{n}=r^{*}+E(\dot{q})+\rho
$$

donde:

\section{$r^{n} \quad$ Tasa de interés real neutral \\ $r^{*} \quad$ Tasa de interés real externa \\ $E(\dot{q})$ Expectativa de depreciación real de la moneda local \\ $q \quad$ Logaritmo natural del tipo de cambio real \\ $\rho \quad$ Indicador de premio por riesgo soberano}

De acuerdo con este enfoque, la tasa de interés interna en términos reales debería converger en estado estacionario a la tasa de interés de paridad internacional.

12 Se denota con $\dot{q}$ como la tasa de crecimiento de una variable $z$ entre $t y \mathrm{t}-1$. 


\section{Modelo semiestructural con componentes no observables}

Laubach y Williams $(2003,2015)$ y Holston, Laubach y Williams (2016) aproximan la tasa de interés real neutral para la economía estadounidense, mediante un modelo macroeconómico compuesto por seis ecuaciones, de acuerdo con el cual la dinámica de la economía se puede representar a partir de una curva IS que describe el equilibrio en el mercado de bienes y servicios; $y$ una curva de Phillips que recoge la dinámica inflacionaria. Estas dos corresponden, respectivamente, a las siguientes ecuaciones de señal:

$$
\begin{aligned}
& \left(y_{t}-y_{t}^{p}\right)=\sum_{s=1}^{S} \alpha_{s}^{y}\left(y_{t-s}-y_{t-s}^{p}\right)+\sum_{\nu=1}^{V} \alpha_{v}^{r}\left(r_{t-\nu}-r_{t-\nu}^{n}\right)+\chi_{1, t}^{\prime} \alpha+\varepsilon_{t}^{y} \\
& \left(\pi_{t}-\pi_{t}^{M}\right)=\sum_{p=1}^{P} \beta_{p}^{\pi}\left(\pi_{t-p}-\pi_{t-p}^{M}\right)+\sum_{q=1}^{Q} \beta_{q}^{y}\left(y_{t-q}-y_{t-q}^{p}\right)+\chi_{2, t}^{\prime} \beta+\varepsilon_{t}^{\pi}
\end{aligned}
$$

donde:

$y_{t}$ logaritmo natural del $P I B$

$y_{t}^{p} \quad$ logaritmo natural del PIB potencial

$r_{t} \quad$ tasa de política monetaria real

$\pi_{t}^{M}$ meta de inflación

$\chi_{1, t}^{\prime}$ otras variables que explican la brecha del producto

$\chi_{2, t}^{\prime}$ otras variables que explican la inflación

$\varepsilon_{t}^{y, \pi}$ proceso ruido blanco con media cero y variancia constante

Para completar el modelo suponen que el producto potencial crece a una tasa que sigue una caminata aleatoria:

$$
\begin{gathered}
y_{t}^{p}=y_{t-1}^{p}+\dot{y}_{t}^{p}+\varepsilon_{t}^{y^{p}} \\
\dot{y}_{t}^{p}=\dot{y}_{t-1}^{p}+\varepsilon_{t}^{\dot{y}^{p}}
\end{gathered}
$$

donde:

$\dot{y}^{p} \quad$ tasa de crecimiento del producto potencial

$\varepsilon_{t}^{\dot{y}^{p}} \quad$ término de error media cero y variancia constante

La dinámica de la tasa de interés real neutral se relaciona con el crecimiento del producto potencial.

$$
r_{t}^{n}=c y_{1}^{p}+z_{1}
$$

donde:

$z \quad$ otros factores que determinan la tasa real neutral 
Estos otros factores que explican la tasa de interés real neutral se estiman a partir de los errores que resultan de estimar la ecuación (6) por mínimos cuadrados ordinarios y posteriormente se modelan como un proceso $\mathrm{AR}(1)$.

$$
Z_{t}=\delta Z_{t-1}+\varepsilon_{t}^{z}
$$

El proceso de estimación mediante el filtro de Kalman requiere de valores iniciales para los parámetros del modelo y para las variables de estado. Para proveer al modelo con estos valores iniciales de los parámetros, se recurre a estimar las ecuaciones de señal por mínimos cuadrados ordinarios. Para el caso de las ecuaciones de estado que requieren valores iniciales para las variables no observables (producto potencial y tasa real neutral), se realizó una aproximación mediante el filtro de Hodrick y Prescott sobre las series PIB y tasa de interés real observadas ${ }^{13}$.

La principal fortaleza de esta metodología de aproximación a la TIRN es que incorpora la estructura básica de la economía, además de que se trata de un modelo parsimonioso.

\section{Tendencia estocástica común implícita}

Magud y Tsounta (2012) obtienen una estimación de la TIRN que parte del supuesto de que la curva de rendimiento -específicamente el margen entre la tasa de corto y la de largo plazo- podría ser informativo sobre la postura de política monetaria en un país. Esta idea fue modelada por Basdevant, Bjorksten y Karagedikli (2004), mediante una tendencia estocástica común entre las tasas nominales de largo plazo y de corto plazo. Magoud y Tsounta proponen el siguiente modelo en forma estado-espacio ${ }^{14}$.

$$
\begin{aligned}
& R_{t}^{c p}=r_{t}^{n}+\pi_{t+12}^{e}+\varepsilon_{t}^{1} \\
& R_{t}^{l p}=r_{t}^{n}+\pi_{t+12}^{e}+\alpha_{t}+\varepsilon_{t}^{2} \\
& r_{t}^{n}=r_{t-1}^{n}+\vartheta_{t}^{1} \\
& \alpha_{t}=\mu_{0}+\mu_{1} \alpha_{t-1}+\vartheta_{t}^{2}
\end{aligned}
$$

donde:

$R^{c p}$ tasa nominal de corto plazo

$R^{l p}$ tasa nominal de largo plazo

$\mathrm{p}^{e}$ expectativas de inflación a 12 meses

$a_{t}$ premio por riesgo

En este modelo, la tasa de interés de corto plazo es igual a la suma de la TIRN, las expectativas de inflación a 12 meses y una perturbación estocástica. Por su parte, la tasa de interés de largo plazo consta de la suma de la tasa de interés de corto plazo, de un premio por riesgo y una perturbación estocástica.

Las siguientes dos ecuaciones son de transición, para las que se supone una caminata aleatoria para la TIRN real $y$ un proceso AR(1) estacionario para el premio por riesgo. Todas las perturbaciones se suponen independientes e idénticamente distribuidas (i.i.d.) con media cero y varianza constante. El modelo se estima mediante el filtro de Kalman.

13 Se verificó que las series $y_{t}$ y $Z_{t}$ son estacionarias. El valor de lambda utilizado fue de 2250, siguiendo los resultados de Segura y Vásquez (2011).

14 Se ajustó la nomenclatura original de variables que emplearon Magud yTsounta (2012) para mantener la coherencia a lo largo del presente documento de investigación. 


\section{Reglas de Taylor dinámicas}

Magud y Tsounta (2012) también proponen dos especificaciones de la regla de Taylor para estimar la TIRN. La primera es un modelo para la TIRN expresada en términos nominales, en la que la tasa de política nominal es una función de las desviaciones de la inflación respecto a la meta del banco central y del producto respecto a su nivel potencial (brecha del producto). Si ambas brechas son cero, la tasa de interés debería establecerse en el nivel de la TIRN. El modelo consta de una ecuación de estado para la tasa de interés de corto plazo y dos ecuaciones de transición:

$$
\begin{aligned}
& R_{t}^{c p}=r_{t}^{n}+\pi_{t+1}^{e}+\beta\left(\pi_{t}-\pi_{t}^{*}\right)+\theta y_{t}^{b}+\varepsilon_{t}^{1} \\
& R_{t}^{l p}=r_{t}^{*}+\pi_{t+1}^{e}+\alpha+\varepsilon_{t}^{2} \\
& r_{t}^{n}=r_{t-1}^{n}+g_{t-1} \\
& g_{t}=g_{t-1}+\vartheta_{t}^{1}
\end{aligned}
$$

El proceso de transición es una caminata aleatoria para $g_{t}$, definida como la tasa de variación para la TIRN expresada en términos nominales. De nuevo, todas las perturbaciones se suponen i.i.d. con media cero $y$ variancia constante, $y$ el modelo se estima mediante filtro de Kalman.

La segunda especificación de regla de Taylor es aumentada por expectativas de inflación. En este caso, la TIRN es ahora una tasa real y se incluye una ecuación para la tasa de interés nominal de largo plazo, con la misma dinámica de transición que en el modelo previo:

$$
\begin{aligned}
& R_{t}^{c p}=R_{t}^{n}+\beta\left(\pi_{t}-\pi_{t}^{M}\right)+\theta y_{t}^{b}+\varepsilon_{t}^{1} \\
& R_{t}^{n}=R_{t-1}^{n}+g_{t-1} \\
& g_{t}=g_{t-1}+\vartheta_{t}^{1}
\end{aligned}
$$

\section{VAR estructural}

Brzoza-Brzezina (2002) propone un método de estimación de la TIRN mediante un VAR identificado (estructural) basado en la imposición de restricciones de largo plazo a la Blanchard y Quah (1989). Empiezan por definir la tasa de interés real como la suma de la TIRN y una brecha de tasas:

$$
r_{t}=r_{t}^{n}+r_{t}^{b}
$$

Y luego supone que la tasa de interés real neutral y la brecha de tasas siguen procesos autorregresivos estacionarios:

$$
\begin{aligned}
& r_{t}^{n}=\Phi_{1}(L) r_{t-1}^{n}+\mu_{1 t}=\Xi_{1}(L) \mu_{1 t} \\
& r_{t}^{b}=\Phi_{2}(L) r_{t-1}^{b}+\mu_{2 t}=\Xi_{2}(L) \mu_{2 t}
\end{aligned}
$$

donde $\Xi_{1}(L)$ y $\Xi_{2}(L)$ son polinomios de rezagos tales que $\Xi(L)=(I-\Phi(L) L)^{-1}$. Esto implica que la tasa de interés puede expresarse en términos de las perturbaciones básicas:

$$
r_{t}=\Xi_{1}(L) u_{1 t}+\Xi_{2}(L) u_{2 t}
$$


Cuando la tasa de interés se desvía de su nivel neutral, esto tiene en un efecto en el nivel de inflación. Brzoza-Brzezina supone que el cambio en la inflación es una fracción $\Psi$ de la brecha de tasas:

$$
\Delta \pi_{t}=\Psi\left(r_{t}^{b}\right)=\Psi\left[\Xi_{2}(L)\right] u_{2 t}
$$

De esta forma, tanto el cambio en la inflación como la tasa de interés pueden expresarse como un sistema de rezagos distribuidos de las perturbaciones básicas:

$$
\left[\begin{array}{c}
\Delta \pi_{t} \\
r_{t}
\end{array}\right]=\left[\begin{array}{ll}
S_{11}(L) & S_{12}(L) \\
S_{21}(L) & S_{22}(L)
\end{array}\right]\left[\begin{array}{c}
u_{1 t} \\
u_{2 t}
\end{array}\right]
$$

Para recuperar las perturbaciones básicas, primero se estima un VAR sin restricciones:

$$
\left[\begin{array}{c}
\Delta \pi_{t} \\
r_{t}
\end{array}\right]=\left[\begin{array}{ll}
A_{11}(L) & A_{12}(L) \\
A_{21}(L) & A_{22}(L)
\end{array}\right]\left[\begin{array}{c}
\Delta \pi_{t-1} \\
r_{t-1}
\end{array}\right]+\left[\begin{array}{c}
\varepsilon_{1 t} \\
\varepsilon_{2 t}
\end{array}\right]
$$

con representación de medias móviles dada por:

$$
\left[\begin{array}{c}
\Delta \pi_{t} \\
r_{t}
\end{array}\right]=\left[\begin{array}{ll}
C_{11}(L) & C_{12}(L) \\
C_{21}(L) & C_{22}(L)
\end{array}\right]\left[\begin{array}{c}
\varepsilon_{1 t} \\
\varepsilon_{2 t}
\end{array}\right]
$$

y cuyas perturbaciones son una función de las perturbaciones estructurales:

$$
\left[\begin{array}{l}
\varepsilon_{1 t} \\
\varepsilon_{2 t}
\end{array}\right]=\left[\begin{array}{ll}
s_{11}(L) & s_{12}(L) \\
s_{21}(L) & s_{22}(L)
\end{array}\right]\left[\begin{array}{l}
u_{1 t} \\
u_{2 t}
\end{array}\right]
$$

Así, las perturbaciones básicas se podrían calcular a partir de los residuos $\boldsymbol{\varepsilon}_{\boldsymbol{t}}$ del VAR no restringido si se conocieran los coeficientes $S_{i j}(0)$. Para ello, Brzoza-Brzezina impone las siguientes restricciones:

i- $\quad$ Las variancias de las perturbaciones básicas son iguales 1.

ii- $S_{11}(1)=0$ en el sistema original. Esta restricción de largo plazo se impone en vista de que la perturbación $u_{1 t}$ no afecta $\Delta \pi_{t}$.

iii- Dado que la política monetaria opera con rezago, se hace $S_{12}(0)=0$ para indicar que la brecha de tasas no tiene efecto contemporáneo sobre la inflación.

A partir de estas restricciones y de una estimación de la matriz de variancias y covariancias de las preturbaciones $\varepsilon_{t}$, los restantes coeficientes $S_{i j}(0)$ se pueden obtener de la siguiente manera:

$$
\begin{aligned}
& s_{11}(0)={ }_{-}^{+} \sqrt{\operatorname{var}\left(\varepsilon_{1 t}\right)} \\
& s_{21}(0)={ }_{-}^{+}\left[\frac{C_{11}(1)}{C_{12}(1)}\right] \sqrt{\operatorname{var}\left(\varepsilon_{1 t}\right)} \\
& s_{22}(0)=\sqrt{-2 \frac{s_{21}(0)}{s_{11}(0)} \operatorname{cov}\left(\varepsilon_{1 t}, \varepsilon_{2 t}\right)+s_{21}^{2}(0)+\operatorname{var}\left(\varepsilon_{2 t}\right)}
\end{aligned}
$$


Así, la TIRN puede calcularse como el resultado únicamente de perturbaciones estructurales:

$$
r_{t}^{n}=S_{21}(L) u_{1 t}
$$

En la siguiente sección se describe la información utilizada para estimar la TIRN a partir de las metodologías expuestas en este apartado.

\section{Datos utilizados}

El cuadro 3 del Anexo presenta el detalle de las series de datos utilizadas en este estudio, así como sus fuentes. La periodicidad es mensual en todos los casos y se considera información desde enero-2002 a junio-2016, aunque el período de estimación varía en función de la metodología empleada.

La serie de datos de la tasa de política monetaria merece especial consideración. Como se ha mencionado, antes de 2004, el BCCR no contaba con una tasa explícita de política monetaria, sino que los intermediarios financieros consideraban la tasa de los Bonos de Estabilización Monetaria a seis meses como una referencia para establecer sus tasas activas y pasivas. Como se documenta en Tenorio (2008), es en febrero de 2004 cuando se define una TPM. Esta tasa ha sido modificada cualitativamente en cinco ocasiones desde entonces: progresivamente pasó de ser una tasa pasiva a 30 días a una tasa activa a un día. Es en junio del 2011, cuando se define la TPM como el valor central de un corredor de tasas en el Mercado integrado de liquidez (MIL).

Con el objetivo de disponer de una tasa de interés de política coherente para un período con extensión, tal que permita el análisis cuantitativo, Castro y Chaverri (2013) definen un indicador que refleja la postura de la política del Banco Central para el período enero 1999 a mayo del $2011^{15}$ (cuando aún no existía una clara definición de TPM). En este estudio, se emplea la TPM efectivamente anunciada por el BCCR para el período de junio 2011 en adelante, y de enero 2009 a mayo 2011 el indicador definido por Castro y Chaverri (2013) ${ }^{16}$.

En términos generales, Castro y Chaverri (2013) infieren el valor de la tasa de interés a un día plazo en una curva de rendimientos construida a partir de la información de tasas de interés de los instrumentos que el Banco Central utilizó para realizar absorción monetaria a diferentes plazos. Posteriormente, concluyen que existe una alta correlación entre este indicador y la tasa de los Bonos de Estabilización Monetaria a seis meses plazo, considerada en su momento como la referencia para el resto de tasas del sistema financiero. El resultado se muestra en el gráfico 1.

GRÁFICO 1

\section{COSTA RICA: TASA DE POLÍTICA MONETARIA}

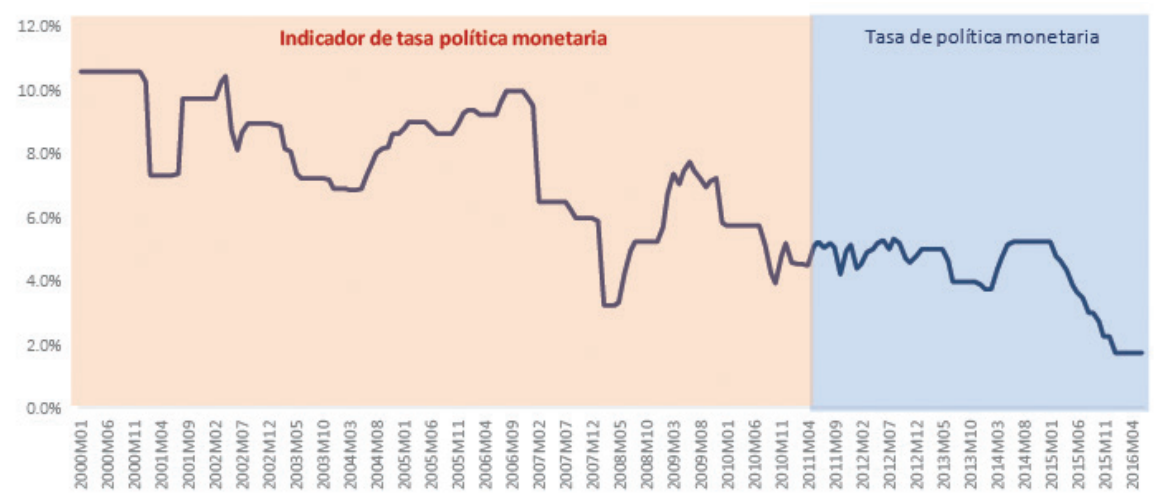

Fuente: Elaboración propia con base en información de Castro y Chaverri (2013) y BCCR.

15 Serie de tiempo que los autores facilitan a solicitud de quien esté interesado.

16 Solo en el caso del modelo semiestructural, se decidió iniciar en el año 2006. 


\section{RESULTADOS}

El cuadro 1 presenta las estimaciones de la TIRN para Costa Rica, obtenidas mediante las metodologías detalladas en la sección anterior. Los resultados difieren pero guardan coherencia entre sí. El promedio simple de las estimaciones (período común enero-2009 a mayo-2015) es 1,4\%, una cifra levemente inferior, pero comparable a la obtenida por Segura y Vindas (2012), el FMI y la OCDE, si bien el rango de valores es más amplio (0,6\% a 3\%). Debe acotarse que la estimación mediante paridad de tasas de interés muestra una volatilidad muy alta comparada con el resto de estimaciones y por ende se decidió excluirla.

CUADRO 1

COSTA RICA: ESTIMACIÓN DE LA TIRN

\begin{tabular}{lcc}
\hline Método & Periodo & Promedio \\
\hline Modelo semiestructural & $2006-2016$ & $0,6 \%$ \\
Regla de Taylor dinámica & $2009-2015$ & $1,2 \%$ \\
Regla de Taylor dinámica aumentada por expectativas & $2009-2016$ & $3,0 \%$ \\
Tendencia estocástica común & $2009-2016$ & $1,2 \%$ \\
\hline
\end{tabular}

Fuente: Elaboración propia.

Como se aprecia en el gráfico 2, las estimaciones obtenidas muestran una evolución similar. Se omite graficar la estimación mediante paridad de tasas de interés, puesto que la mayor parte del período 2009-2016 presenta valores negativos.

GRÁFICO 2

COSTA RICA: ESTIMACIONES DE LA TIRN 2009-2015

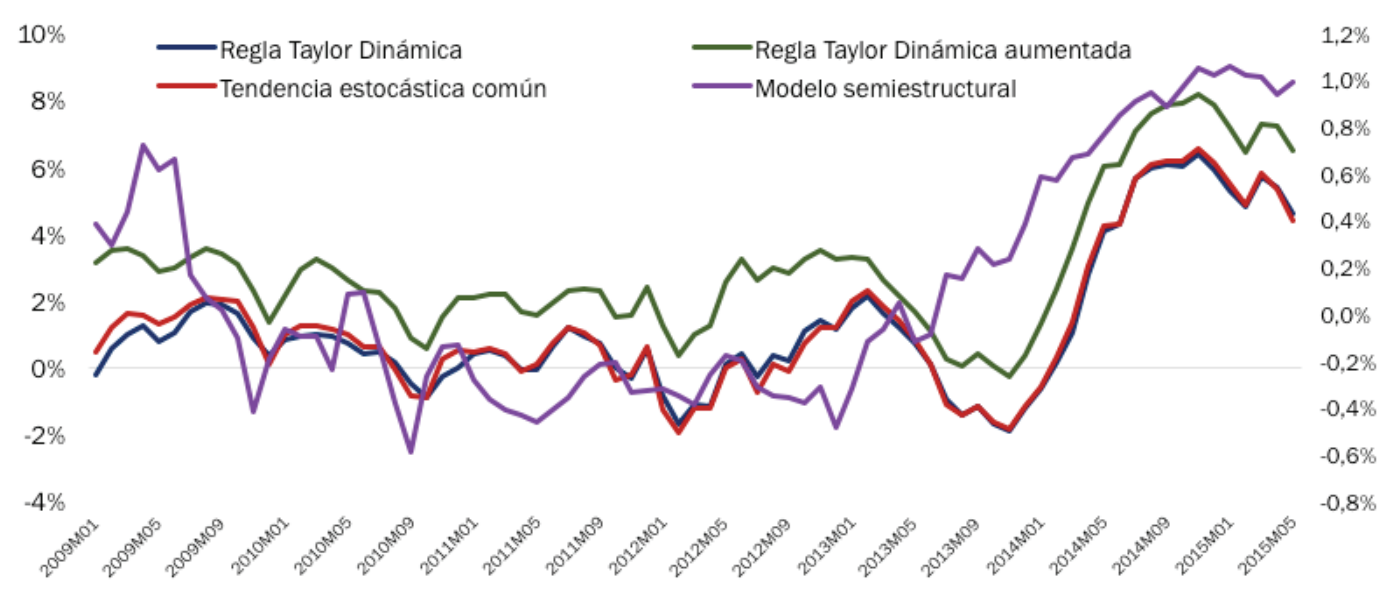

Fuente: Elaboración propia. 
Como se ha repetido, la importancia de contar con una estimación adecuada de la tasa de interés real neutral radica en que permite obtener una guía sobre el estado de la política monetaria del banco central, al analizar la brecha de tasa de interés.

De acuerdo con una regla de política tipo Taylor, si el Banco Central prevé presiones de demanda (brecha del producto positiva), deberá ajustar al alza su tasa de política en una magnitud tal que la brecha de tasas sea positiva, de manera que este aumento de la tasa de interés real tenga un efecto contractivo sobre la demanda agregada, reduciendo la brecha del producto. Lo anterior sugiere que la relación entre la brecha de tasas de interés y la brecha del producto es negativa y que movimientos en la brechas de tasas deberían preceder movimientos en la brecha de producto.

Una forma de verificar la fuerza de dicha relación en la práctica $y$, por ende, de evaluar la utilidad de las estimaciones de la TIRN como guía sobre las decisiones de política, es analizar los comovimientos entre ambas brechas. Para ello, en el gráfico 3 se presenta las correlaciones móviles de la brecha de tasas con la brecha del producto, para varios rezagos (valores negativos del eje horizontal) $y$ adelantos (valores positivos).

Dos resultados confirman la relación esperada esbozada en el párrafo anterior: primero, la correlación más alta entre las brechas es negativa (-0.51); y segundo, los movimientos en la brecha de tasas de interés adelantan a los movimientos en la brecha del producto, pues dicha correlación más alta se presenta en el rezago 4. Debe resaltarse que este número de períodos de adelanto de la brecha de tasas de interés con respecto a la brecha del producto es coherente con el que se contempla en la ecuación de la curva IS, dentro del Modelo macroeconómico de proyección trimestral que utiliza el Banco Central de Costa Rica.

\section{GRÁFICO 3 \\ CORRELACIONES ENTRE BRECHA DE TASAS DE INTERÉS \\ Y BRECHA DE PRODUCTO ADELANTOS Y REZAGOS}

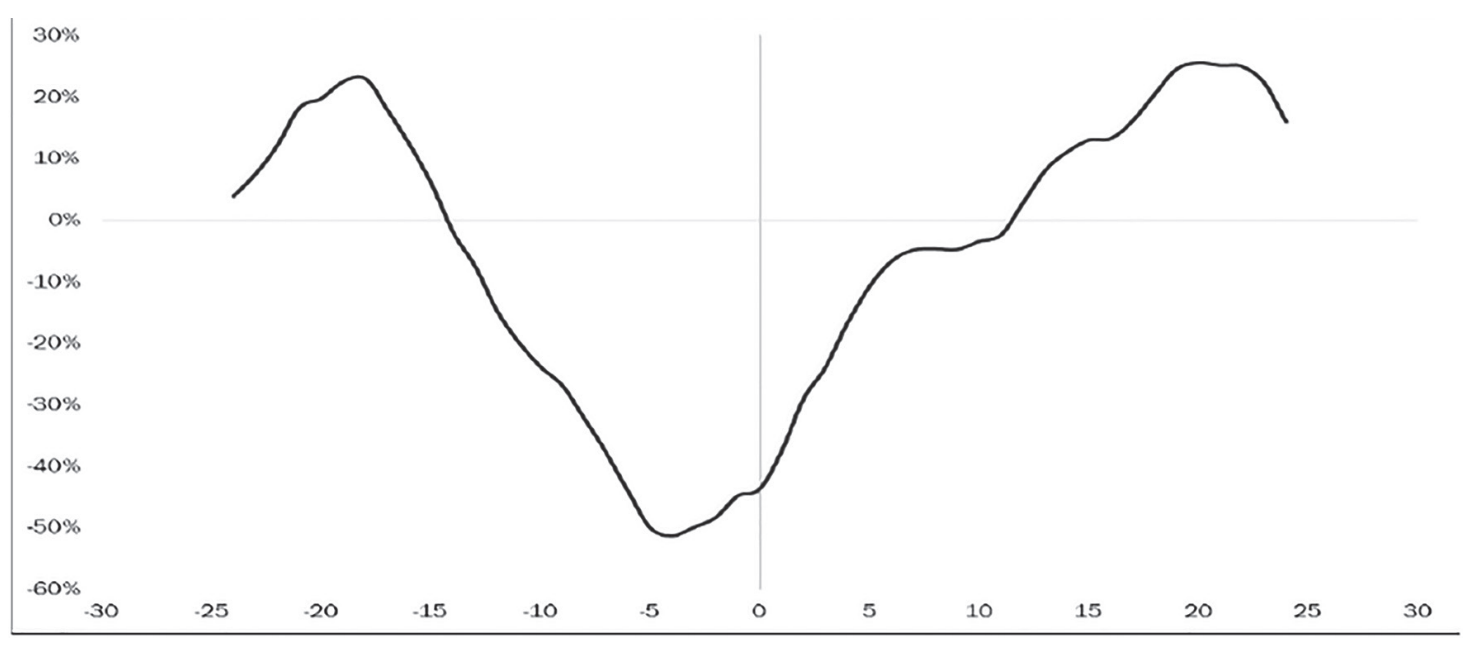

Fuente: Elaboración propia.

Como un resultado adicional proveniente de la estimación del modelo semiestructural propuesto por Laubach y Williams $(2003,2015)$, se obtiene una estimación de la tasa de crecimiento potencial para la economía costarricense que para el período 2003-2015 se ubica en torno a 4,2\% ${ }^{17}$.

17 De acuerdo con el procedimiento que sigue el BCCR para estimar el producto potencial, este primer resultado debe combinarse con el proveniente de la estimación de una función de producción tipo Cobb-Douglas, y con filtros estadísticos. La combinación se realiza con métodos bayesianos. 


\section{CONCLUSIONES}

Para un banco central cuyo objetivo es el control de la inflación y que utiliza una tasa de política monetaria como instrumento, resulta crucial contar con una adecuada estimación de la tasa de interés real neutral, para poder evaluar el efecto probable de cambios en dicho instrumento. Sin embargo, la tasa de interés real neutral no es constante en el tiempo, lo cual hace necesario que los bancos centrales actualicen periódicamente sus estimaciones de dicha variable no observable.

En el caso de la economía costarricense, una limitación que enfrenta para la estimación de esta variable es el período relativamente corto durante el cual el BCCR ha seguido una estrategia de control monetario mediante una tasa de interés de política. Para solventar este problema, en el pasado se recurrió a utilizar una tasa pasiva promedio del sistema financiero, bajo el supuesto de que reflejaba la intención de política del BCCR en períodos, cuando esta entidad realizaba su política mediante la colocación de títulos llamados Bonos de Estabilidad Monetaria a un plazo promedio de 6 meses.

En este estudio se estimó la TIRN para Costa Rica mediante varias metodologías con fundamentos teóricos. Se incorporó por primera información de la Tasa de Política Monetaria (junto con un indicador de TPM para el período previo a junio 2011).

El promedio simple de las estimaciones sugiere que la TIRN para Costa Rica se ubica alrededor de $1,4 \%{ }^{18}$. La evolución de la brecha de la tasa de interés resultante de esta estimación de la TIRN se asocia de manera inversa con la evolución de la brecha del producto: en general, posturas de política monetaria expansivas (contractivas) se han asociado con mayores (menores) presiones de demanda futuras. La magnitud y la antelación de esta asociación son compatibles con los hechos estilizados implícitos en el modelo de proyección de mediano plazo utilizado por el BCCR.

18 Este valor, sumado a la meta de inflación de 3\%, sugiere una tasa de interés nominal neutral de 4,4\%. 


\section{REFERENCIAS}

Amato, J. (2005). The Role of the Natural Rate of Interest in Monetary Policy. (BIS Working Papers171). Obtenido de http://www.bis.org/publ/work171.pdf

Banco Central de Costa Rica. (Diciembre de 2011). Informe de Inflación. Obtenido de http://www.bccr. fi.cr/publicaciones/politica_monetaria_inflacion/Informe_Inflacion_Diciembre_2011.pdf

Banco Central de Costa Rica. (2016). Programa Macroeconómico 2017-2018. San José, Costa Rica. Obtenido de http://www.bccr.fi.cr/publicaciones/politica_monetaria_inflacion/PM_2017-2018.pdf

Basdevant, O., Bjorksten, N., \& Karagedikli, O. (2004). Estimating a Time Varying Neutral Real Interest Rate for New Zeland. Wellington: Reserve Bank of New Zealand(Discussion Paper No. 2004/01).

Blanchard, O., \& Quah , D. (1989). The Dynamic Effects of Aggregate Demand and Supply Disturbances. American Economic Review, 79(4), 655-673.

Brzoza-Brzezina, M. (2002). Estimating the Natural Rate of Interest: A SVAR Approach (Working Papers, No. 27). Banco Nacional de Polonia.

Brzoza-Brzezina, M. (2006). The Information Content of the Neutral Rate of Interest. The Economics of Transition, 14(2), 391-412. https://doi.org/10.1111/j.1468-0351.2006.00255.x

Castro, A., \& Chaverri, C. (2013). Indicador de Tasa de política monetaria del Banco Central de Costa Rica (DT-11-2013). Banco Central de Costa Rica. Departamento de Investigación Económica. Obtenido de http://www.bccr.fi.cr/investigacioneseconomicas/ politicamonetariaeinflacion/Indicador_TPM_del_BCCR.pdf

Fisher, I. (1911). The purchasing power of money: its determination and relation to credit, interest and crises. Nueva York: The Macmillan Co.

Fondo Monetario Internacional. (2016). Costa Rica. Selected issues and analytical notes (IMF Country Report No. 16/132). Washington, D.C. Obtenido de https://www.imf.org/en/ Publications/CR/Issues/2016/12/31/Costa-Rica-Selected-Issues-and-Analytical-Notes-43918

Fuentes, R. (2008). La tasa de interés real neutral: definiciones y evidencia para las economías latinoamericanas. En J.Torres, R. Fuentes, J. Carrera, \& T. N. da Silva Filho (Eds.), Estimación y uso de variables no observables en la región (pp. 135-144). México D.F.: Centro de Estudios Monetarios Latinoamericanos.

Galí, J. (2002). New Perspectives on Monetary Policy, Inflation, and the Business Cycle. (NBER Working Paper No. 8767). https://doi.org/10.3386/w8767

Holston, K., Laubach, T., \& Williams, J. C. (2016). Measuring the Natural Rate of Interest: International Trends and Determinants (Federal Reserve Bank of San Francisco Working Paper 2016-11). San Francisco, CA. Obtenido de http://www.frbsf.org/economic-research/files/ wp2016-11.pdf

Keynes, J. M. (1930). A Treatise on Money. Londres: Harcourt, Brace and company.

Keynes, J. M. (1936). The General Theory of Employment, Interest and Money. Cambridge: Macmillan Cambridge University Press.

Laubach, T., \& Williams, J. C. (2003). Measuring the Natural Rate. The Review of Economics and Statistics, 85(4), 1063-1070. https://doi.org/10.1162/003465303772815934 
Laubach, T., \& Williams, J. C. (2015). Measuring the Natural Rate of Interest Redux (Federal Reserve Bank of San Francisco Working Paper 2015-16). San Francisco, CA.

Ley 7558. (3 de noviembre de 1995). San José, Costa Rica. Obtenido de http://www.pgrweb.go.cr/ scij/Busqueda/Normativa/Normas

Magud, N., \& Tsouta, E. (2012). To Cut or Not to Cut? That is the (Central Bank's) Question. In Search of the Neutral Interest Rate in Latin America (WP/12/243). Washington: Fondo Monetario Internacional.

Muñoz, E., \& Tenorio, E. (2007). Estimación de la tasa de interés real neutral para Costa Rica: 1991-2006 (Documento de Investigación DIE-04-2007-DI). Banco Central de Costa Rica. Departamento de Investigación Económica. Obtenido de http://www.bccr.fi.cr/ investigacioneseconomicas/politicamonetariaeinflacion/Estimacion_tasa_interes_real_ neutral_Costa_Rica,periodo_1991-2006.pdf

Organización para la Cooperación y el Desarrollo Económico. (2016). OECD Economic Surveys: Costa Rica 2016: Economic Assessment. París: OECD Publishing. https://doi.org/10.1787/ eco_surveys-cri-2016-en

Segura, C., \& Vásquez, J. P. (2011). Estimación del parámetro de suavizamiento del filtro HodrickPrescott para Costa Rica. Banco Central de Costa Rica. Obtenido de http://www.bccr. fi.cr/investigacioneseconomicas/metodoscuantitativos/Estimacion_del_parametro_de_ suavizamiento_del_filtro_de_Hodrick_y_Prescott_para_CR.pdf

Segura, C., \& Vindas, A. (2012). Estimación de la tasa de interés real neutral y la tasa natural de desempleo para la economía costarricense. Banco Central de Costa Rica (Documento de Investigación. DI-07-2012). Banco Central de Costa Rica. Departamento de Investigación Económica. Obtenido de http://www.bccr.fi.cr/investigacioneseconomicas/ politicamonetariaeinflacion/2016-DI-05-Costa\%20Rica_TasaIntersRealNeutral.pdf

Taylor, J. B. (1993). Discretion versus Policy Rules in Practice. Carnegie-Rochester Conference Series on Public Policy, 39(Supplement C) 195-214. https://doi.org/10.1016/01672231(93)90009-L

Tenorio, E. (2008). Recopilación de definición y cambios en la Tasa de política monetaria del Banco Central de Costa Rica (Informe Técnico DIE-037-2008-IT). Banco Central de Costa Rica. Obtenido de http://www.bccr.fi.cr/investigacioneseconomicas/politicamonetariaeinflacion/ Recopilacion_definicion_cambios_tasa_politica_monetaria_Banco_Central_CR.pdf

Vásquez, J. P., \& Segura, C. (2011). Estimación del parámetro de suavizamiento del filtro de Hodrick y Prescott para Costa Rica (Documento de Investigación DEC-DIE-DT 006-2011). Departamento de Investigación Económica, Banco Central de Costa Rica.

Wicksell, K. (1938). Interest and Prices (R.F. Kahn, Trad.). Londres: Macmillan.

Woodford, M. (2003). Interest and Prices: Foundations of a Theory of Monetary Policy. Princeton, N.J.: Princeton University Press. 
ANEXO

CUADRO 2

COSTA RICA: ESTIMACIONES DE TASA DE INTERÉS REAL NEUTRAL

\begin{tabular}{|c|c|c|c|c|}
\hline & $\begin{array}{c}\text { Muñoz y } \\
\text { Tenorio } \\
(2007)\end{array}$ & $\begin{array}{c}\text { Segura y } \\
\text { Vindas }(2012)\end{array}$ & $\begin{array}{c}\text { FMI } \\
(2016)\end{array}$ & $\begin{array}{l}\mathrm{OCDE}^{\mathrm{a} /} \\
(2016)\end{array}$ \\
\hline Período de estudio & 2001-2006 & $2001-2011$ & Varios & $2008-2015$ \\
\hline Periodicidad & Trimestral & Mensual & Trimestral & Mensual \\
\hline \multicolumn{5}{|l|}{ Metodología } \\
\hline Ad-hoc (observada) & $2,8 \%$ & $2,0 \%$ & & \\
\hline Filtro Hodrick-Prescott & $2,6 \%$ & $1,9 \%$ & & \\
\hline Modelo semiestructural & $3,1 \%$ & $2,2 \%$ & $1,4 \%$ & \\
\hline VAR estructural & & $1,3 \%$ & & \\
\hline Paridad de tasas & $3,1 \%$ & n.d. & $1,6 \%$ & \\
\hline $\begin{array}{l}\text { Regla de Taylor aumentada por } \\
\text { expectativas }\end{array}$ & & & $2,4 \%$ & $1,6 \%$ \\
\hline Modelo de equilibrio general & & & $2,6 \%$ & \\
\hline Modelo monetario & & & $1,8 \%$ & \\
\hline Promedio & $2,9 \%$ & $1,9 \%$ & $1,9 \%$ & $1,6 \%$ \\
\hline
\end{tabular}

a/ Tasa nominal es 4,6\% en la fuente original, se presenta la real tras restar la meta de inflación.

Fuente: Elaboración propia. 
CUADRO 3

DEFINICIÓN DE VARIABLES Y FUENTES

\begin{tabular}{|c|c|c|}
\hline Variable & Datos utilizados & Fuente \\
\hline$R_{t}, R_{t}^{c p}$ & Tasa de política monetaria nominal & Banco Central de Costa Rica \\
\hline$R_{t}^{l p}$ & $\begin{array}{l}\text { Tasa ponderada de bonos de Hacienda a más de } 5 \\
\text { años, mercado secundario }\end{array}$ & $\begin{array}{l}\text { Departamento de Inspección y Análisis de } \\
\text { Mercados e Intermediarios, SUGEVAL }\end{array}$ \\
\hline$r^{*}$ & $\begin{array}{l}\text { Tasa de interés internacional real, libor } 6 \text { meses } \\
\text { deflatada con la inflación de EE.UU. para } t+12\end{array}$ & Bloomberg \\
\hline$\pi_{t}$ & Variación interanual del Índice de Precios al Consumidor & Instituto Nacional de Estadística y Censos \\
\hline$\pi_{t}^{M}$ & Meta para la tasa de inflación interanual & Banco Cental de Costa Rica \\
\hline$\pi_{t+1}^{e}$ & $\begin{array}{l}\text { Variación interanual del Índice de Precios al } \\
\text { Consumidor en } t+12\end{array}$ & Instituto Nacional de Estadística y Censos \\
\hline$q_{t}$ & $\begin{array}{l}\text { Tipo de cambio efectivo real multilateral con } \\
\text { ponderadores móviles, logaritmo natural }\end{array}$ & Banco Central de Costa Rica \\
\hline$r_{t}$ & Embi Costa Rica & Bloomberg \\
\hline$\alpha_{t}$ & Margen de bonos 2020 del Gobierno de Costa Rica. & Bloomberg \\
\hline$\alpha$ & Promedio de para el período de estimación & \\
\hline$y$ & Producto Interno Bruto, colones constantes de 1991. & Uso interno, Banco Central de Costa Rica \\
\hline$y^{b}$ & $\begin{array}{l}\text { Diferencia porcentual del PIB real respecto a su nivel } \\
\text { potencial (Hodrick-Prescott, ) }\end{array}$ & Estimación propia. \\
\hline
\end{tabular}

Fuente: Elaboración propia.

Todos los derechos reservados. Universidad de Costa Rica. Este artículo se encuentra licenciado con Creative Commons Reconocimiento-NoComercial-SinObraDerivada3.0 Costa Rica. Para mayor información escribir a revista.iice@ucr.ac.cr 
